\begin{tabular}{ccc}
\hline & International Journal of Engineering \& Technology, 7 (2.32) (2018) $470-472$ \\
SPC & Website: www.sciencepubco.com/index.php/IJET \\
Research paper & Technology \\
\hline
\end{tabular}

\title{
Effective Bug Triage With Software Reliability
}

\author{
B.Chaitanya Krishna ${ }^{1}$, N.Sai Prasanna ${ }^{2}$, B.Hema Lahari ${ }^{3}$, Ch.Ajay ${ }^{4}$ \\ ${ }^{1,2,3,4}$ Dept of CSE, K L E F, Vaddeswaram, India \\ *Corresponding author E-mail: chaitu2502@kluniversity.in
}

\begin{abstract}
Programming associations spend in excess of 45 percent of cost in overseeing programming bugs. An inevitable progress of settling bugs is bug triage, which wants to precisely dole out a planner to another bug. To reduce the time cost in manual work, content portrayal frameworks are associated with coordinate customized bug triage. In this paper, we address the issue of data diminishment for bug triage, i.e., how to diminish the scale and upgrade the idea of bug data. We unite case assurance with feature decision to at the same time decrease data scale on the bug estimation and the word estimation. To choose the demand of applying event assurance and feature decision, we expel characteristics from evident bug instructive records and create a judicious model for another bug enlightening file. We precisely investigate the execution of data diminish on completely 600,000 bug reports of two significant open source wanders, particularly Eclipse and Mozilla. The results exhibit that our data abatement can satisfactorily decrease the data scale and improve the precision of bug triage. Our work gives an approach to manage using techniques on data taking care of to outline diminished and stunning bug data in programming change and upkeep.
\end{abstract}

Keywords:data mining, content characterization.

\section{Introduction}

Everything considered, data mining (once in a while called data or learning presentation) is the course toward segregating data from exchange perspectives and amassing it into basic information information that can be used to make pay, cuts costs, or both. Data mining creating PC programs is one of different honest to goodness gadgets for researching data. It draws in customers to segregate data from an expansive combination of estimations or focuses, sort it, and graph the affiliations saw. Everything considered, data mining is the path toward finding affiliations or cases among different fields in basic social databases.

\subsection{Driving/Retail:}

Data mining empowers publicizing relationship to assemble models in light of recorded data to foresee who will respond to the new pushing endeavors, for instance, post office based mail, electronic indicating exertion... et cetera. Through the results, showcasing masters will have reasonable approach to manage direct pitch strong things to ran customers. Data mining passes on a basic measure of focal concentrations to retail affiliations moderately as progressing. Through market compartment examination, a store can have a fitting creation plot in a way that customers can buy visit getting things together with marvelous. In like manner, it other than engages the retail relationship to offer certain refunds for particular things that will pull in more customers.

\subsection{Spare/Banking}

Data mining gives cash related affiliations information about affect information and credit isolating. By building a model from recorded customer's data, the bank and budgetary establishment can pick impossible and appalling advances. Also, data mining empowers banks to see false MasterCard trades to secure charge card's proprietor.

\subsection{Social occasion}

By applying information mining in operational building information, makers can see faulty sorts of mechanical assembly and pick consummate control parameters. For instance semiexecutive makers has a test that even the states of storing up conditions at various wafer creation plants are commensurate, the nature of wafer are disperse same and some for obscure reasons even has surrenders. Information mining has been applying to pick the degrees of control parameters that affect the generation of amazing wafer. By then those immaculate control parameters are utilized to make wafers with required quality.

\subsection{Governments}

Information mining helps government office by burrowing and taking a gander at records of cash related exchange to create plans that can perceive impose shirking or criminal exercises.

\subsection{Law Endorsement}

Information mining can help law specialists in seeing criminal suspects and what's all the more discovering these lawbreakers by looking in zone, terrible conduct shape propensity, and differing instances of practices. 


\subsection{Experts}

Information mining can help specialists by reviving their information looking at process; in this manner, engaging those more prominent opportunity to oversee differing attempts.

\section{Related Work}

Web content setback 's and reshaped strongly made webpage pages are major blunders, and they really impact the convenience of Web applications. Current contraptions for website page support can't deal with the proficiently conveyed pages that are omnipresent on the present Internet. We demonstrate a dynamic test age technique for the space of dynamic Web applications. The technique uses both joined cement and delegate execution and conveys state indicate checking. The structure produces tests typically, runs the tests getting certified goals on wellsprings of information, and limits the conditions on the duties regarding coming up short tests with the target that the resulting bug reports are about nothing and critical in finding and settling the covered shortcomings. Our mechanical get together Apollo finishes the system for the PHP programming language. Apollo produces test responsibilities for a Web application, screens the application for crashes, and supports that the yield consents to the HTML detail. This paper demonstrates Apollo's calculations and execution, and an exploratory evaluation that uncovered 673 blames in six PHP Web applications. A key gathering masterminded put for something change meanders is the bug report vault. Despite the way that its use can redesign the thing change process in various ways, reports added to the store should be triaged. A triager picks whether a report is imperative. Basic reports are then managed for mix into the task's progress system.

To help triggers with their work, this article shows a machine adjusting way to deal with oversee make recommenders that help with an assortment of choices went for streamlining the progress method. The recommenders made with this approach are right; for example, recommenders for which fashioner to name a report that we have impacted utilizing along these lines to manage have accuracy in the area of $70 \%$ and $98 \%$ more than five open source meanders. As the framework of a recommender for a specific errand can require critical exertion and be grim, we in like way demonstrate a way to deal with oversee help the setup of such recommenders that fundamentally chops down the cost of setting up a recommender for a meander. We demonstrate that recommenders for which designer should settle a bug can be immediately arranged with this approach and that the coordinated recommenders are inside $15 \%$ precision of handtuned fashioner recommenders.

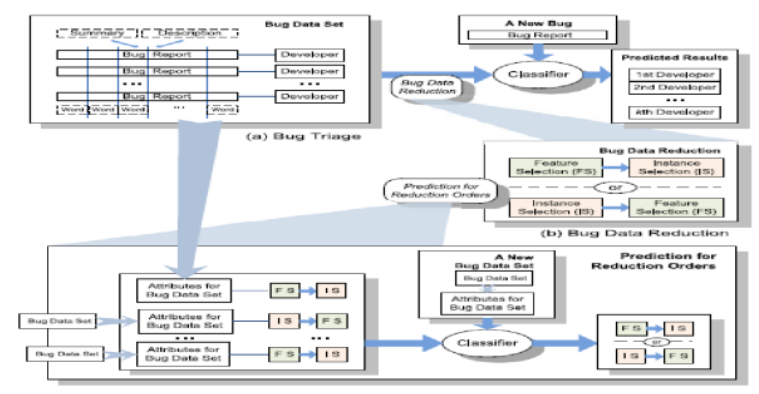

Fig1. System architecture

\section{System Analysis}

\subsection{Existing System}

to investigate the relationship in bug information, Sandusky et al. shape a bug report system to evaluate the reliance among bug reports.

Other than assessing relationship among bug reports, Hong et al. make a modeler pleasant relationship to look at the joint effort among engineers in setting of the bug information in Mozilla meander. This virtuoso social association is immense to welcome the maker gathering and the errand progress. by mapping bug needs to engineers, Xuan et al. see the draftsman prioritization in open source bug vaults. The fashioner prioritization can see engineers and help endeavors in programming upkeep.To take a gander at bug information, Zimmermann et al. configuration studies to fashioners and clients in three open sources meanders. In light of the examination of audits, they portray what impacts an OK bug to report and set up a classifier to see whether the probability of a bug report ought to be progressed.

Duplicate bug reports debilitate bug information by yielding the cost of managing bugs. To see copy bug reports, Wang et al. design a trademark dialect directing methodology by sorting out the execution data.

\subsection{Weights of Existing System}

1. Traditional programming examination isn't completely sensible for the sweeping scale and complex information in programming stories.

2. In customary programming change, new bugs are physically triaged by a star shape, i.e., a human triager. Because of the tremendous number of especially asked for bugs and the nonattendance of predominance of the essential number of bugs, manual bug triage is costly in time cost and low in accuracy.

\subsection{Proposed System:}

1. In this paper, we address the issue of information lessening for bug triage, i.e., how to reduce the bug information to spare the work cost of originators and invigorate the quality to help the course of action of bug triage.

2. Data reducing for bug triage expects to produce a little scale and overwhelming game-plan of bug information by expelling bug reports and words, which are excess or nonlighting up.

3. In our work, we consolidate existing strategy for example confirmation and highlight choice to meanwhile lessen the bug estimation and the word estimation. The diminished bug information contain less bug reports and less words than the central bug information and give relative data over the fundamental bug information. We review the lessened bug information as indicated by two criteria: the cross of a lighting up party and the precision of bug triage.

4. In this paper, we propose a sharp model to pick the request of applying occasion choice and highlight choice. We interpret such articulation as measure for diminishing solicitations.

5. Drawn on the encounters in programming metrics, 1 we expel the properties from recorded bug edifying records. By at that point, we set up a twofold classifier on bug enlight- 
ening files with separated qualities and expect the request of applying occasion choice and highlight request for another bug educational social event.

\subsection{Motivations Driving Interest Of Proposed System}

Experimental works out as anyone might expect show that applying the case affirmation system to the illuminating social affair can diminish bug reports yet the exactness of bug triage might be decreased. Applying the part affirmation structure can reduce words in the bug information and the precision can be extended.

Meanwhile, joining the two strategies can create the accuracy, what's more lessen bug reports and words. In context of the traits from unquestionable bug enlightening collections, our sharp model can give the precision of 71.8 percent for exact retribution the diminishment sort out.

We show the issue of information decrease for bug triage. This issue expects to develop the instructive saving of bug triage in two perspectives, to be specific a) to in the meantime decrease the sizes of the bug estimation and the word estimation and b) to refresh the accuracy of bug triage.

We propose a blend way to deal with oversee manage looking out for the issue of information lessening. This can be seen as a use of occasion choice and highlight choice in bug stores.

We collect a twofold classifier to expect the request of applying occasion choice and highlight choice. To the degree anyone is concerned, the request of applying occasion choice and highlight choice has not been broke down in related spaces.

\section{Experimental Results}

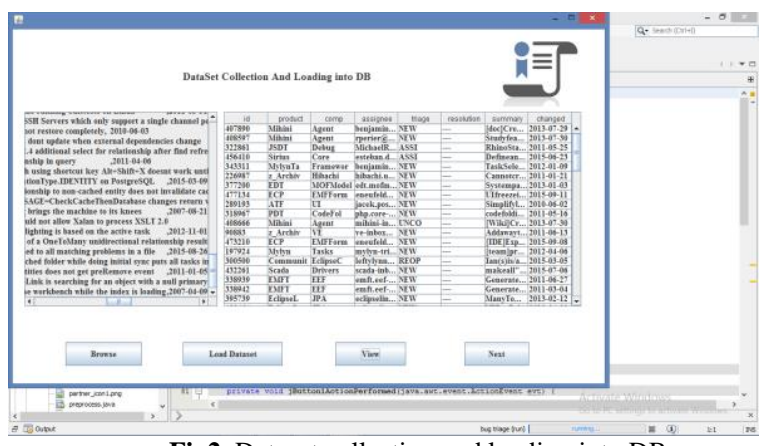

Fig2. Dataset collection and loading into DB

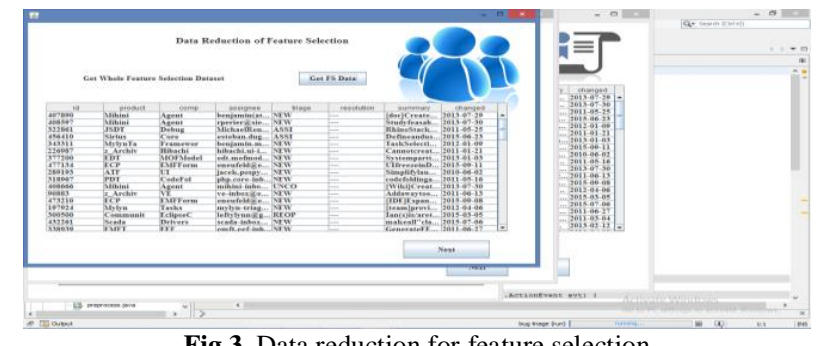

Fig 3. Data reduction for feature selection

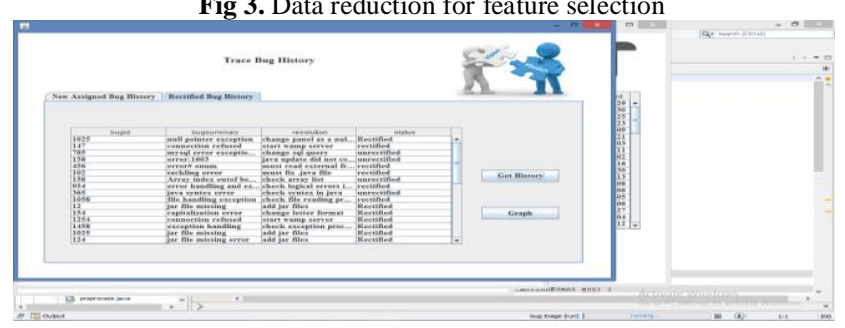

Fig4. Traced Bug History

\section{Conclusion}

Bug triage is an over the top advance of programming support in both work cost and time cost. In this paper, we join set attestation with occasion choice to lessen the measure of bug enlightening collections and besides update the information quality. To pick the request of applying occasion choice and highlight attestation for another bug instructive chronicle, we tie characteristics of each bug illuminating record and set up a farsighted model in setting of genuine enlightening accumulations. We likely take a gander at the information decreasing for bug triage in bug stores of two sweeping open source twists, particularly Eclipse and Mozilla. Our work gives a way to deal with oversee administer utilizing system on information preparing to plot diminished and decision bug information in programming progression and support. In future work, we imagine re-designing the put off consequences of information diminishment in bug triage to explore how to set up a stunning bug lighting up once-finished and handle a space particular programming undertaking. For foreseeing diminishment orders, we need to pay tries to locate the potential association between the properties of bug lighting up get-togethers and the decreasing sales.

\section{References}

[1] J. Anvik, L. Hiew, and G. C. Murphy, Who should settle this bug? in Proc. 28th Int. Conf. Softw. Eng., May 2006, pp. 361- 370.

[2] S. Artzi, A. Kie_zun, J. Dolby, F. Tip, D. Passage, A. Paradkar, and M. D. Ernst, Discovering bugs in web applications utilizing dynamic test age and unequivocal state show checking, IEEE Softw., vol. 36, no. 4, pp. 474-494, Jul./Aug. 2010.

[3] J. Anvik and G. C. Murphy, Reducing the exertion of bug report triage: Recommenders for advancement constructed choices, ACM Trans. Delicate. Eng. Methodol., vol. 20, no. 3, article 10, Aug. 2011.

[4] C. C. Aggarwal and P. Zhao, Towards graphical models for content administering, Knowl. Illuminate. Syst., vol. 36, no. 1, pp. 1-21, 2013.

[5] Bugzilla, (2014). [Online

[6] K. Balog, L. Azzopardi, and M. de Rijke, Formal models for master finding in big business corpora, in Proc. 29th Annu. Int. ACM SIGIR Conf. Res. Create. Educate. Recovery, Aug. 2006, pp. $43-$ 50 .

[7] P. S. Bishnu and V. Bhattacherjee, Programming deficiency forecast utilizing quad tree-based $\mathrm{k}$-implies grouping calculation, IEEE Trans. Knowl. Information Eng., vol. 24, no. 6, pp. 1146-1150, Jun. 2012.

[8] H. Brighton and C. Mellish, Advances in occasion determina-tion for example based learning calculations, Data Mining Knowl. Revelation, vol. 6, no. 2, pp. 153-172, Apr. 2002.

[9] S. Breu, R. Premraj, J. Sillito, and T. Zimmermann, Data needs in bug reports: Improving collaboration amongst engineers and clients, in Proc. ACM Conf. Comput. Upheld Cooperative Work, Feb. 2010, pp. 301-310.

[10] V. Bol_on-Canedo, N. S_anchez-Maro no, and A. AlonsoBetanzos, An audit of highlight determination techniques on manufactured information, Knowl. Educate. Syst., vol. 34, no. 3, pp. 483- 519, 2013.

[11] V. Cerver_on and F. J. Ferri, Another push toward the base predictable subset: A tabu hunt way to deal with the dense closest neighbor lead, IEEE Trans. Syst., Man, Cybern., Part B, Cybern., vol. 31, no. 3 , pp. $408-413$, Jun. 2001. 